\title{
Descripción de un modelo de gestión de enfermedad en una población en diálisis en Colombia
}

\author{
Alfonso Bunch ${ }^{1}$, Layla Tamer ${ }^{2}$, Freddy Ardila ${ }^{1}$, Stefano Laganis ${ }^{1}$, Ricardo Castaño ${ }^{1}$, \\ Jasmin Vesga ${ }^{1}$, Patricia López ${ }^{1}$, Mauricio Sanabria ${ }^{1}$

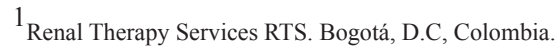

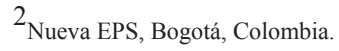

\section{Resumen}

Introducción: Los Modelos de Gestión de Enfermedad (MGE) permiten identificar riesgos y priorizar intervenciones para lograr mejores desenlaces clínicos. Este trabajo tiene por objetivo describir un MGE diseñado para una población en diálisis e identificar los factores de riesgo cardiovascular y metabólico en dicha población.

Métodos: Se describe el MGE. Se incluyeron pacientes mayores de 18 años, prevalentes en diálisis entre el $1^{\circ}$ de marzo y el 31 de agosto de 2013; se evaluaron variables demográficas y clínicas basales, y se identificaron factores de riesgo según la presencia de diabetes. Para el análisis se usó estadística descriptiva en Stata ${ }^{\circledR} 12$.

Resultados: Se analizaron 2219 pacientes, 1004 (45.24\%) de ellos eran diabéticos, 624 (28.1\%) tuvieron antecedentes de enfermedad cardiovascular, en 990 (94.37\%) se reportó alguna alteración en el ecocardiograma, siendo la más frecuente la cardiopatía hipertensiva en el $58.63 \%$. Se observó que del total de los pacientes en hemodiálisis, 271 (13\%), y en diálisis peritoneal $112(5 \%)$ tuvieron mal control de la volemia, PCR elevada en el $64.4 \%$ y TSH $>10 \mathrm{UI} / \mathrm{ml}$ en el $12.1 \%$ de los sujetos. El índice tobillo-brazo (ITB) se encontró alterado en el $37.5 \%$ de los diabéticos, versus $21.8 \%$ en los no diabéticos ( $\mathrm{p}<0.001)$; se observaron diferencias significativas en cuanto a sobrepeso, $40.03 \%$ versus $26.91 \%$, en diabéticos y no diabéticos, respectivamente.

Conclusiones: Este modelo de gestión de enfermedad basado en gestoría de casos e integración de redes de servicios puede ser una respuesta de alto impacto para poblaciones en diálisis con importante comorbilidad.

Palabras clave: Factores de riesgo cardiovascular, Diálisis, Enfermedad renal crónica terminal, Gestión de enfermedad (fuente DeCS).

\section{Description of a disease management model in patients undergoing dialysis in Colombia}

\section{Abstract}

Introduction: The disease management models (DMM) allows to identify risks and prioritize interventions to achieve better outcomes. This work aims to describe a DMM designed for a population undergoing dialysis and identify the cardiovascular and metabolic risk factors. 
Methods: We made a description of a DMM; this is a cross sectional study inpatients, older than 18 years, prevalent in dialysis between March 1st and August 31st, 2013. Demographic and clinical variables were assessed at baseline and we identified risk factors according to the presence of diabetes. Descriptive statistics was implemented for the analysis.

Results: We evaluated 2219 patients, 1004 (45.24\%) were diabetics, 624 (28.1\%) had history of cardiovascular disease, in 990 (94.37\%) report some alteration in the echocardiogram, hypertensive heart disease being the most common in $58.63 \%$. We found that $271(13 \%)$ hemodialysis patients and $112(5 \%)$ in peritoneal dialysis had poor volemic control; high C-reactive protein in $64.4 \%$ and thyroid stimulating hormone greater than $10 \mathrm{IU} / \mathrm{ml}$ in $12.1 \%$ of subjects. The ankle-brachial index was abnormal in $37.5 \%$ of diabetics versus $21.8 \%$ non-diabetics $(p<0.001)$, were found significant differences in overweight $40.03 \%$ versus $26.91 \%$ $(\mathrm{p}<0.001)$ in diabetic and non-diabetic respectively.

Conclusion: This disease management model based in cases managers and integration of services networks could be a high impact response for populations undergoing dialysis with important comorbidity.

Key words: Cardiovascular Risk Factors, Dialysis, End Stage Renal Disease, Disease Management (MeSHsource).

\section{Introducción}

$\mathrm{E}$ 1 paciente con Enfermedad Renal Crónica (ERC) en terapia dialítica representa un reto para los sistemas de salud en tanto que, a la complejidad del tratamiento dialítico y de la enfermedad de base, se suman una serie de complicaciones, especialmente de origen cardiovascular'; esta condición se traduce en mayor demanda de servicios y, en consecuencia, en mayores costos para el sistema de salud.

A la alta comorbilidad de los pacientes en diálisis contribuyen la presencia de los factores de riesgo cardiovascular tradicionales, como la hipertensión, la diabetes, la dislipidemia, los desórdenes del metabolismo mineral, el mal control de la volemia, $\mathrm{y}$ aquellos no tradicionales o los llamados nuevos factores de riesgo, como la inflamación crónica, la disfunción endotelial, la Proteína C Reactiva (PCR) aumentada, la hiperhomocisteinemia, el hipotiroidismo clínico y subclínico ${ }^{2-8}$. Además, empeora el panorama para los pacientes en diálisis la mayor presencia de Enfermedad Arterial Periférica (EAP), asociada con riesgo elevado de enfermedad coronaria, accidente cerebro vascular y enfermedad cardiovascular $^{9,10}$. Todo lo anterior conlleva a que en esta población la frecuencia de hospitalizaciones con estancias más prolongadas sea significativamente alta, especialmente por causa cardiovascular ${ }^{11,12}$.

En respuesta a la complejidad del manejo que requieren estos pacientes, se han propuesto mode- los de gestión de enfermedad como una estrategia integral y costo-eficiente para alcanzar mejores resultados en salud ${ }^{13-16}$; adicionalmente, en poblaciones geográficamente dispersas y que reciben servicios no siempre integrados en sus diferentes niveles de atención, los modelos de gestión de enfermedad se podrían complementar con la filosofía de las Redes Integradas de Sistemas de Salud, lanzada por la Organización Panamericana de la Salud (OPS) ${ }^{17}$ y la gestoría de casos, estrategias que ya han demostrado su eficacia ${ }^{18-20}$. En los modernos sistemas de salud, la innovación en modelos que entreguen cuidado médico de manera más integral, más eficiente y con desenlaces de excelencia para el paciente y la sociedad, son una ruta hacia el mejoramiento de las condiciones de vida de las poblaciones.

El objetivo de este trabajo es documentar el diseño de un modelo de gestión de enfermedad implementado en una población con Enfermedad Renal Crónica (ERC) grado 5 en diálisis crónica y describir los factores de riesgo cardiovascular y metabólicos identificados en estos pacientes.

\section{Métodos}

Este Modelo de Gestión de Enfermedad se diseñó a partir de una revisión de la literatura y del análisis de los reportes del comportamiento clínico anual de morbimortalidad de la población en diálisis perte- 
neciente a la Entidad Promotora de Salud (EPS) del régimen contributivo Nueva $\mathrm{EPS} \AA$, atendidos en la red de Renal Therapy Services (RTS)®; se describió el modelo en sus tres fases de implementación. Se utilizó un diseño de corte transversal para realizar la caracterización del riesgo basal de los pacientes, el cual incluyó sujetos mayores de 18 años, en diálisis crónica con más de 90 días en terapia y con, al menos, tres meses de permanencia en RTS. El estudio se realizó durante el período comprendido entre el $1^{\circ}$ de marzo y el 31 de agosto de 2013 . Las variables demográficas y clínicas se obtuvieron de la historia clínica de RTS (RENIR®).

Las variables incluidas para análisis fueron: edad, sexo, tiempo en diálisis, modalidad de diálisis, causa de la ERC, antecedentes de Enfermedad Cardiovascular (ECV), índice de masa corporal (IMC), índice tobillo brazo (ITB), colesterol, albúmina, hemoglobina, fósforo, kt/v, Proteína C Reactiva, TSH, hemoglobina glicosilada (HbA1c). También se registraron variables relacionadas con calidad de diálisis, incluidas en la cuenta de alto costo en Colombia ${ }^{21}$, como fueron: albúmina, hemoglobina, fósforo, y kt/v en HD y kt/v en DP. Se realizó evaluación ecocardiográfica al subgrupo de pacientes con enfermedad cardiovascular, agrupando los resultados como cardiopatía hipertensiva, cardiopatía isquémica, valvulopatías, Fracción de Eyección (FE) menor del 50\%, Presión Sistólica de la Arteria Pulmonar (PSAP) mayor de $25 \mathrm{mmHg}$ y normal. El mal control volémico se definió clínicamente como un porcentaje de ganancia interdialítica mayor de $5 \%$ en los pacientes en hemodiálisis (HD) y como la sumatoria de la ultrafiltración (UF) y la diuresis menor a $750 \mathrm{cc} /$ día en los pacientes en diálisis peritoneal (DP). Todas las variables fueron tomadas al inicio del seguimiento.

Para el análisis descriptivo se utilizaron medianas y medias con sus respectivas medidas de dispersión para las variables continuas, además de porcentajes para las variables categóricas. El análisis estadístico se realizó en Stata ${ }^{\circledR}$ 12. Para todas las pruebas de hipótesis se usaron dos colas y niveles de significación del 5\%. La ejecución del presente estudio fue aprobada y monitorizada por un comité de ética institucional.

\section{Resultados}

Descripción del Modelo: El Modelo de gestión de Enfermedad fue diseñado en 3 fases: 1) caracterización del riesgo, 2) intervención, evaluación y medición de resultados y 3) gestión para el mejoramiento continuo. Cada una de las fases se compone de unas actividades específicas que, en conjunto, buscan la identificación e intervención oportuna de los principales factores de riesgo cardiovascular y metabólico, con el fin de impactar en los desenlaces clínicos definidos como disminución de eventos de hospitalización y estancias hospitalarias.

Durante la primera fase, todos los pacientes reciben información acerca de las intervenciones que se les realizarán, de los beneficios esperados con el modelo, y de sus deberes y derechos como pacientes; también reciben educación en diabetes, manejo y aplicación de insulina, control de la volemia y talleres de adherencia farmacológica. De manera paralela, se inicia la identificación de los factores de riesgo para la estratificación y priorización de las intervenciones a realizar en la siguiente fase del modelo.

En la segunda fase, se realiza la intervención de los pacientes, de acuerdo a la caracterización del riesgo; el modelo incluye las valoraciones médicas especializadas de mayor demanda, como son endocrinología, cardiología y cirugía vascular; durante esta fase se lleva a cabo el seguimiento a las hospitalizaciones.

Para la adecuada ejecución de esta fase, el modelo cuenta con unos pilares fundamentales que son: la educación del paciente y su familia, la gestión de la información clínica y la integración de redes de servicio. Los gestores de casos son los impulsores y garantes del desarrollo del modelo, son el concepto integrador de redes de servicios, gestionan día a día la información relacionada con la estratificación del riesgo de los pacientes, las necesidades de interconsultas y remisiones, los eventos de hospitalización y las solicitudes de exámenes complementarios fuera de la unidad renal; para ello cuentan con herramientas automatizadas de información que permiten un proceso ágil de seguimiento y toma de decisiones.

En la tercera fase, se analizan los resultados y se implementan procesos de mejoramiento continuo de cara a alcanzar metas propuestas. La figura 1 esque- 
matiza el MGE basado en gestoría de casos e integración de redes de servicios.

Caracterización del riesgo: Se evaluaron 2219 pacientes que fueron atendidos en 48 unidades renales de 13 departamentos de Colombia. De ellos, 1306 $(58.85 \%)$ eran hombres, la edad tuvo una media de $62.93(\mathrm{DE}=14.7)$ años, 1267 pacientes $(57.1 \%)$ estaban en HD al inicio y 952 (42.9\%) en DP, 1004 (45.24\%) eran diabéticos, la mediana del tiempo en terapia al inicio fue mayor para los no diabéticos: $4.75(\mathrm{RIC}=3.4)$ años, versus $2.61(\mathrm{RIC}=6.1)$ años. En la tabla 1 se describen las características demográficas y clínicas al inicio del seguimiento.

Un subgrupo de 1049 pacientes fue evaluado con estudio ecocardiográfico, de los cuales 990 (94.37\%) reportaron alguna alteración; en 172 (16.39\%) se identificó cardiopatía isquémica y en 615 (58.62\%) se identificó cardiopatía hipertensiva. En la tabla 2 se presentan los hallazgos ecocardiográficos discriminados por presencia o no de diabetes.
En relación con otros factores de riesgo, se encontró que del total de los pacientes en HD, 271(13\%) tuvieron mal control volémico y 112 (5\%) en DP; el porcentaje de pacientes con ITB alterado en diabéticos versus no diabéticos fue significativamente diferente: 377 (37.54\%), frente a 265 (21.81\%), respectivamente, $(\mathrm{p}<0,001)$. También se observaron diferencias significativas en las proporciones de pacientes con sobrepeso: 402 (40.03\%), contra 327 (26.91\%), entre diabéticos y no diabéticos, respectivamente. Los otros factores de riesgo comparados no mostraron diferencias significativas (tabla 3).

\section{Discusión}

El Modelo de Gestión de Enfermedad para una población con ERC grado 5 en diálisis aquí descrito tiene como piedras angulares la gestoría de casos y la integración de redes de servicios, y representa una

\section{Figura 1}

Modelo de gestión de enfermedad con gestoría de casos e integración de redes de servicios

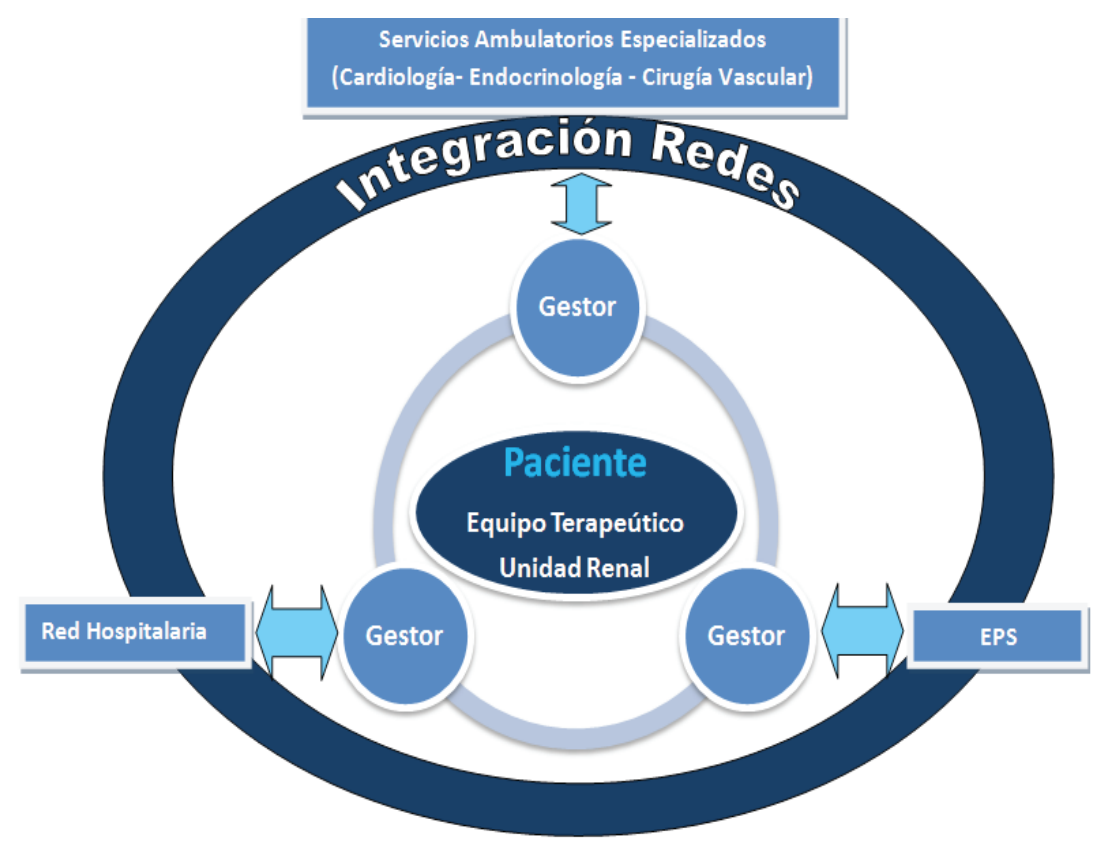




\section{Tabla 1}

Características demográficas y clínicas de los pacientes al inicio del estudio según presencia o no de diabetes.

\section{Características}

Edad [media;DE] en años

Sexo; hombres [n;\%]

Tiempo en diálisis [mediana;RIQ] añosa

Modalidad; Diálisis peritoneal [n;\%]

Acceso Vascular [n;\%]b

Fistula arteriovenosa

Catéter vascular

Causa de la ERC [n;\%]

Hipertensión arterial

Diabetes

Enfermedad glomerular

Obstructiva

Desconocida

Otras

Índice de masa corporal [media;DE] en $\mathrm{kg} / \mathrm{m} 2$

Colesterol total [media;DE] en mg/dl

Colesterol LDL [media;DE] en mg/dl

Albúmina [media;DE] en mg/dl

Hemoglobina [media;DE] en mg/dl

Fósforo [media;DE] en mg/dl

$\mathrm{kt} / \mathrm{v}$ en diálisis peritoneal [media;DE]

$\mathrm{Kt} / \mathrm{v}$ en hemodiálisis [media;DE]

HbAlc [n;\%] en \%c

$\mathrm{HbA} 1 \mathrm{c}<=6.5 \%$

$\mathrm{HbAlc}>6.5 \mathrm{y}<=7.5 \%$

HbAlc $>7.5$ y $<=8 \%$

$\mathrm{HbA} 1 \mathrm{c}>8 \%$

Antecedente ECV [n;\%]

a RIQ: Rango Intercuartílico.

b Dato disponible en hemodiálisis.

c Hemoglobina glicosilada disponible para 995 diabéticos.

\section{Diabéticos (n 1004)}

$67(10.59)$

572(56.97)

2.61 (3.4)

486(48.40)

403(77.80)

115(22.20)

76(7.56)

$860(85.65)$

$6(0.59)$

12(1.19)

24(2.39)

26(2.58)

25.99(5.77)

$181.80(53.63)$

101.79(43.22)

$3.79(0.56)$

11.7(1.76)

4.69(1.38)

2.22(0.61)

$1.50(0.37)$

321(32.26)

292(29.34)

121(12.16)

261(26.23)

$359(35.75)$

\begin{tabular}{ll}
$\begin{array}{l}\text { No Diabéticos } \\
\text { (n 1215) }\end{array}$ & $\begin{array}{l}\text { Total } \\
\text { (n 2219) }\end{array}$ \\
\hline $60(16.7)$ & $62.93(14.7)$ \\
$734(60.41)$ & $1306(58.85)$ \\
$4.75(6.1)$ & $3.55(5.53)$ \\
$466(21.0)$ & $952(42.9)$
\end{tabular}

575(76.77)

978(44.07)

174(23.23)

289(13.02)

546(44.93)

622(28.03)

$860(38.75)$

186(15.30)

192(8.65)

78(6.41)

90(0.40)

201(16.54)

225(10.13)

204(16.79)

230(10.36)

23.73(4.22)

25(5.06)

185.7(48.84)

184(51.07)

101.87(39.09)

105(41.34)

3.95(0.52)

$4(0.54)$

$11.62(1.84)$

12(1.80)

4.72(1.57)

$5(1.49)$

2.19(0.70)

2.2(0.66)

1.53(0.30)

$1.52(0.33)$

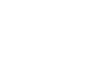


Tabla 2

Hallazgos ecocardiográficos discriminados por presencia o no de diabetes.

\begin{tabular}{|llll|}
\hline Hallazgo & Diabéticos (n 524) & $\begin{array}{l}\text { No Diabéticos } \\
\text { (n 525) }\end{array}$ & Total (n 1049) \\
\hline Cardiopatía Hipertensiva [n;\%] & $306(58.39)$ & $309(58.85)$ & $615(58.62)$ \\
Cardiopatía Isquémica [n;\%] & $106(20.22)$ & $66(12.57)$ & $172(16.39)$ \\
Valvulopatía Leve a Moderada [n;\%] & $30(5.72)$ & $60(11.42)$ & $90(8.57)$ \\
Valvulopatía Severa [n;\%] & $15(3.05)$ & $29(5.52)$ & $44(4.19)$ \\
Fracción de Eyección $<50 \%$ [n;\%] & $32(6.10)$ & $28(5.33)$ & $60(5.71)$ \\
PSAP>25mmHg [n;\%]a & $4(0.76)$ & $5(0.72)$ & $9(0.85)$ \\
Normal [n;\%] & $31(5.91)$ & $28(5.33)$ & $59(5.62)$ \\
\hline a Presión sistólica de la arteria pulmonar. & & & \\
\hline
\end{tabular}

\begin{tabular}{|c|c|c|c|}
\hline \multicolumn{4}{|c|}{ Tabla 3} \\
\hline \multicolumn{4}{|c|}{ Factores de riesgo según presencia o no de diabetes. } \\
\hline Factores de Riesgo & Diabéticos (n 1004) & $\begin{array}{l}\text { No Diabéticos } \\
\text { (n 1215) }\end{array}$ & $\mathbf{P}$ \\
\hline Mal control volémico $[\mathrm{n} ; \%]$ & $150(14.94)$ & 233(19.17) & 0.20 \\
\hline Proteína $C$ reactiva $>3[\mathrm{n} ; \%] \mathrm{mg} / \mathrm{L}$ & $580(57.76)$ & 719(59.17) & 0.46 \\
\hline $\mathrm{TSH}>=10[\mathrm{n} ; \%]$ en $\mathrm{ml} \mathrm{U} / \mathrm{La}$ & $124(13.5)$ & $121(9.95)$ & 0.44 \\
\hline ITB $<0.9$ y $>1.4[n ; \%] 2$ & $377(37.54)$ & $265(21.81)$ & $<0.0001$ \\
\hline $\mathrm{IMC}>=30 \mathrm{~kg} / \mathrm{m} 2[\mathrm{n} ; \%] 3$ & $148(14.74)$ & $87(7.16)$ & 0.10 \\
\hline IMC $>=25-29.99 \mathrm{~kg} / \mathrm{m} 2[\mathrm{n} ; \%] 3$ & $402(40.03)$ & $327(26.91)$ & $<0.0001$ \\
\hline $\mathrm{IMC}<18.5 \mathrm{~kg} / \mathrm{m} 2[\mathrm{n} ; \%] 3$ & $26(0.25)$ & $88(7.24)$ & 0.01 \\
\hline Colesterol Total $>=200 \mathrm{mg} / \mathrm{dl}[\mathrm{n} ; \%]$ & 261(25.99) & $356(29.30)$ & 0.35 \\
\hline Colesterol LDL $>=100 \mathrm{mg} / \mathrm{dl}[\mathrm{n} ; \%]$ & $345(34.36)$ & $494(40.65)$ & 0.06 \\
\hline \multicolumn{4}{|c|}{$\begin{array}{l}\text { a TSH: Hormona estimulante de la tiroides. } \\
\text { b ITB:Índice tobillo brazo dato disponible para } 1730 \text { pacientes. } \\
\text { c IMC: Índice de masa corporal. }\end{array}$} \\
\hline
\end{tabular}

solución en salud para una población con importante comorbilidad, donde casi la mitad padece de diabetes mellitus y, en alrededor de un tercio de ella, se documenta enfermedad cardiovascular importante. El modelo de gestión de enfermedad propone la caracterización del riesgo como una estrategia integral que permite priorizar las intervenciones con el fin de alcanzar mejores resultados en salud.
Aunque al comparar la prevalencia de factores de riesgo entre diabéticos y no diabéticos solo se observaron diferencias importantes en cuanto al ITB e IMC alterados, varios estudios han demostrado el mayor riesgo de complicaciones cardiovasculares en los pacientes diabéticos y los beneficios de la intervención integral ${ }^{22-24}$, en este sentido, adicional al control metabólico, acciones como la medición del 
ITB proveen una oportunidad para la intervención frente al riesgo de eventos cardiovasculares ${ }^{25}$. Por otra parte, el inicio o ajuste del tratamiento a los pacientes en quienes se encontró TSH alta podrá beneficiar la no aparición de fibrilación auricular, tal como lo reportan algunos artículos ${ }^{26}$.

En la tendencia del cuidado preventivo del paciente en diálisis, el reporte anual en Estados Unidos, United States Renal Data System (USRDS), en 2013 evidencia un aumento de $30 \%$ en la toma de ecocardiogramas en el paciente en diálisis en la última década $^{27}$. Es de resaltar que en nuestra población encontramos resultados positivos para enfermedad cardíaca importante en más de un tercio de los sujetos; además, en el 93\% de los casos se identificó algún grado de anormalidad; este porcentaje es superior al observado por otros autores, como en el estudio HEMO que reportó prevalencias de enfermedad cardíaca en pacientes en HD de $80 \%{ }^{28}$. Lo anterior refuerza las recomendaciones internacionales de realizar ecocardiogramas periódicos a los pacientes en diálisis, dado su riesgo cardiovascular incrementado y la alta prevalencia de enfermedad cardíaca en ellos.

El modelo de gestión de enfermedad basado en gestoría de casos e integración de redes de servicios puede ser una respuesta de alto impacto para poblaciones en diálisis con importante comorbilidad, hecho que puede traducirse en mejores desenlaces para los pacientes y eficiencias para el sistema de salud. Estudios posteriores evaluarán el modelo en términos de desenlaces clínicos, costos y calidad de vida de los pacientes.

\section{Conflicto de interés}

Alfonso Bunch, Freddy Ardila, Stefano Laganis, Ricardo Castaño, Jasmin Vesga, Patricia López y Mauricio Sanabria son empleados de RTS Colombia. Layla Tamer es empleada de Nueva EPS.

\section{Contribuciones de los autores}

MS, FA, AB y SL, LT, participaron en el diseño, coordinación del estudio y en el análisis estadístico y generación del manuscrito. JV, PL y RC fueron los responsables de la recolección de los datos, análisis estadístico y contribuyeron en el diseño del estudio y la consolidación del manuscrito. Todos los autores aprobaron el manuscrito final.

\section{Financiación}

El presente estudio fue financiado por un presupuesto para investigación de RTS Colombia.

\section{Agradecimientos}

A los gestores de casos, a los equipos clínicos de RTS y equipo de gestión de Nueva EPS por toda su dedicación en la implementación del Modelo de Gestión de Enfermedad.

\section{Referencias}

1. Levey AS, Beto JA, Coronado BE, Eknoyan G, Foley RN, Kasiske BL, et al. Controlling the epidemic of cardiovascular disease in chronic renal disease: What do we know? What do we need to learn? Where do we go from here? National Kidney Foundation Task Force on Cardiovascular Disease. Am J Kidney Dis. 1998;32(5):853-906.

2. Longenecker JC, Coresh J, Powe NR, Levey AS, FinkNE, Martin A, et al. Traditional cardiovascular disease risk factors in dialysis patients compared with the general population: the CHOICE Study. Clin J Am Soc Nephrol. 2002;13(7):1918-27.

3. Tentori F, Blayney MJ, Albert JM, Gillespie BW, Kerr PG, Bommer J, et al. Mortality risk for dialysis patients with different levels of serum calcium, phosphorus, and PTH: the Dialysis Outcomes and Practice Patterns Study (DOPPS). Am J Kidney Dis. 2008;52(3):519-30.

4. Kalantar K, Regidor D, Kovesdy C, Van D, Horwich T, Fonarrow G. Fluid retention is associated with cardiovascular mortality in patients undergoing long-term hemodialysis. Circulation. 2009;119:671-9.

5. Zoccali C, Mallamaci F, Tripepi G. Traditional and emerging cardiovascular risk factors in end-stage renal disease. Kidney Int Suppl. 2003;63:S105-S10. 
6. Becker BN, Himmelfarb J, Henrich WL, Hakim RM. Reassessing the cardiac risk profile in chronic hemodialysis patients: A hypothesis on the role of oxidant stress and other non-traditional cardiac risk factors. Clin J Am Soc Nephrol. 1997;8(3):475-86.

7. Stenvinkel P, Carrero JJ, Axelsson J, Lindholm B, Heimbürger O, Massy Z. Emerging biomarkers for evaluating cardiovascular risk in the chronic kidney disease patient: how do new pieces fit into the uremic puzzle? Clin J Am Soc Nephrol. 2008;3(2):505-21.

8. Bazeley J, Bieber B, Li Y, Morgenstern H, de Sequera P, Combe C, et al. C-reactive protein and prediction of 1-year mortality in prevalent hemodialysis patients. Clin J Am Soc Nephrol. 2011;6(10):2452-61.

9. Mourad JJ, Cacoub P, Collet JP, Becker F, Pinel JF, Huet D, et al. Screening of unrecognized peripheral arterial disease (PAD) using ankle-brachial index in high cardiovascular risk patients free from symptomatic PAD. J Vasc Surg. 2009;50(3):572-80.

10. Ankle Brachial Index Collaboration, Fowkes FG, Murray GD, Butcher I, Heald CL, Lee RJ, et al. Ankle brachial index combined with Framingham risk score to predict cardiovascular events and mortality. JAMA. 2008;300(2):197208.

11. Collins AJ, Foley RN, Gilbertson DT, Chen SC. The state of chronic kidney disease, ESRD, and morbidity and mortality in the first year of dialysis. Clin J Am Soc Nephrol. 2009;4(Suppl.1):S5-S11.

12. Rayner HC, Pisoni RL, Bommer J, Canaud B, Hecking E, Locatelli F, et al. Mortality and hospitalization in haemodialysis patients in five European countries: results from the Dialysis Outcomes and Practice Patterns Study (DOPPS). Nephrol Dial Transplant. 2009;19(1):108-20.

13. López K, Lorenzo S, Gruss E, Garrido MV, Moreno JA. Aplicación de la gestión por procesos en Nefrología. Gestión del proceso de hemodiálisis. Nefrología. 2002;22(4):329-39.

14. Nissenson AR, Rettig RA. Medicare's end-stage renal disease program: current status and future prospects. Health Aff. 1999;18(1):161-79.

15. Anand S, Nissenson AR. Utilizing a disease management approach to improve ESRD patient outcomes. Semin Dial. 2002;15(1):38-40.

16. Ministerio de la Protección Social, Colombia. Guía De Instrucción Para El Diseño De Programas De Gestión De Enfermedades Crónicas. Observatorio de la Calidad de la Atención en Salud. Dirección General de Calidad [Internet]. 2014 [citado julio de 2014]. Disponible en: http://mps1.minproteccionsocial.gov.co/evtmedica/linea\%2014/presentacion.html

17. López P, Alonso L, García AJ, Fernández IE. Presencia de los atributos para la integración de las redes de servicios de salud. Rev Cubana de Medicina General Integral. 2009;25(4):86-97.

18. Norfolk E, Hartle J. Nephrology Care in a Fully Integrated Care Model: Lessons from the Geisinger Health System. Clin J Am Soc Nephrol. 2013;8(4):687-93.

19. Batres JP, Álvarez M, Gallardo P. De la precisión de cuidados a los cuidados imprescindibles. Las enfermeras gestoras de casos en Andalucía: la enfermera comunitaria de enlace. Rev Adm Sanit. 2009;7(2):313-22.

20. López T, Amoedo ML, Navarro R, Guerrero M. Modelo de gestión de casos en el paciente renal hospitalizado con síndrome sociosanitario. Comunicaciones Presentadas al XXXIII Congreso Nacional. SEDEN [Internet]. 2008 [citado octubre de 2014]. Disponible en: http://www.revistaseden.org/files/1977_128[2].pdf

21. Cuenta de Alto Costo. Situación de la enfermedad renal crónica en Colombia [Internet]. 2014 [citado octubre de 2014]. Disponible en: http://www.cuentadealtocosto.org/

22. Chantrel F, Enache I, Bouiller M, Kolb I, Kunz K, Petitjean P, et al. Abysmal prognosis of patients with type 2 diabetes entering dialysis. Nephrol Dial Transplant. 1999;14(1):129-36.

23. Margolis KL, O'Connor PJ, Morgan TM, Buse JB, Cohen RM, Cushman WC, et al. Outcomes of combined cardiovascular risk factor management strategies in type 2 diabetes: The ACCORD randomized trial. Diabetes care. 2014;37(6):1721-8. 
24. Gæde P, Lund-Andersen H, Parving HH, Pedersen O. Effect of a multifactorial intervention on mortality in type 2 diabetes. N Engl J Med. 2008;358(6):580-91.

25. Lepäntalo M, Fiengo L, Biancari F. Peripheral arterial disease in diabetic patients with renal insufficiency: a review. Diabetes Metab Res Rev. 2012;28(S1):40-5.

26. Cappola AR, Fried LP, Arnold AM, Danese MD, Kuller LH, Burke GL, et al. Thyroid status, cardiovascular risk, and mortality in older adults. JAMA. 2006;295(9):1033-41.

27. U.S. Renal Data System, USRDS 2005 Annual Data Report: Atlas of End-Stage Renal Disease in the United States, National Institutes of Health, National Institute of Diabetes and Digestive and Kidney Diseases, Bethesda, MD [Internet]. 2005 [citado octubre de 2014]. Disponible en: http://www.usrds.org/atlas05.aspx

28. Cheung AK, Sarnak MJ, Yan G, Berkoben M, Heyka R, Kaufman A, et al. Cardiac diseases in maintenance hemodialysis patients: results of the HEMO Study. Kidney int. 2004;65(6):2380-9. 